\title{
Development and validation of nomograms for predicting blood loss in placenta previa with placenta increta or percreta
}

\author{
Ruihui Lu ${ }^{1 \#}$, Ran Chu ${ }^{1 \#}$, Na Gao ${ }^{1}$, Guiyang $\mathrm{Li}^{1}$, Haiyang Tang ${ }^{1}, \mathrm{Xinxin}_{\mathrm{Zhou}}{ }^{1}$, Xiangxin Lan ${ }^{1}$, Shuyi $\mathrm{Li}^{1,2}$, \\ Xi Zhang ${ }^{1}$, Yintao $\mathrm{Xu}^{1}$, Yuyan $\mathrm{Ma}^{1}$ \\ ${ }^{1}$ Department of Obstetrics and Gynecology, Qilu Hospital, Shandong University, Jinan, China; ${ }^{2}$ Department of Radiology, Qilu Hospital, Shandong \\ University, Jinan, China \\ Contributions: (I) Conception and design: R Lu, R Chu, Y Ma; (II) Administrative support: Y Ma; (III) Provision of study materials or patients: X \\ Zhang, Y Xu, Y Ma; (IV) Collection and assembly of data: N Gao, G Li, H Tang, X Zhou, X Lan, S Li; (V) Data analysis and interpretation: R Lu, R \\ Chu; (VI) Manuscript writing: All authors; (VII) Final approval of manuscript: All authors. \\ \#These authors contributed equally to this work. \\ Correspondence to: Yuyan Ma, MD. Department of Obstetrics and Gynecology, Qilu Hospital, Shandong University, 107 Wenhua Xi Road, Jinan, \\ China. Email: yuyanma@sdu.edu.cn.
}

\begin{abstract}
Background: To develop the risk prediction model of intraoperative massive blood loss in placenta previa with placenta increta or percreta.

Methods: This study included 260 patients, of whom 179 were allocated to the development group and 81 to the validation group. Univariate and multivariate logistic regression analyses were used to identify characteristics that were associated with massive blood loss $(\geq 2,500 \mathrm{~mL})$ during cesarean section. A nomogram was constructed based on regression coefficients. Receiver-operating characteristic curve, calibration curve, and decision curve analyses were applied to assess the discrimination, calibration, and performance of the model.

Results: Two models were constructed. The preoperative feature model (model A) consisted of vascular lacunae within the placenta and hypervascularity of the uterine-placental margin, uterine serosa-bladder wall interface, and cervix. The preoperative and surgical feature model (model B) consisted of an emergency cesarean section, no preoperative balloon placement of the abdominal aorta, and the previously mentioned four ultrasound signs. Model B had better discrimination than model A (area under the curve: development group: 0.839 vs. 0.732; validation group: 0.829 vs. 0.736). Model B showed a higher area under the decision curve than model $\mathrm{A}$ in both the training and validation groups.

Conclusions: The preoperative and surgical feature model for placenta previa with placenta increta or percreta can improve the early identification and management of patients who are at high risk of intraoperative massive blood loss.
\end{abstract}

Keywords: Placenta previa; placenta increta; placenta percreta; intraoperative massive blood loss; risk prediction model

Submitted Jul 06, 2020. Accepted for publication Nov 20, 2020.

doi: $10.21037 / \mathrm{atm}-20-5160$

View this article at: http://dx.doi.org/10.21037/atm-20-5160

\section{Introduction}

Over the past 2 decades, several epidemiological studies have shown a direct association between a previous cesarean section and an increased risk of placenta previa and placenta increta or percreta in subsequent pregnancies $(1,2)$. A retrospective Japanese study reported that $37 \%$ of women with placenta previa after previous cesarean sections developed placenta increta or percreta and had an increased risk of fatal maternal complications (3). In China, the 


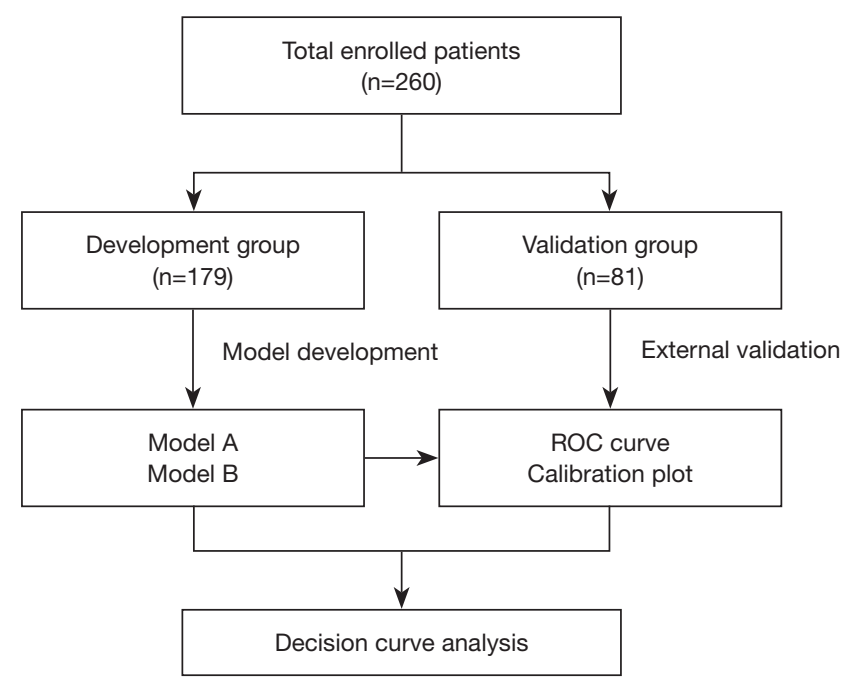

Figure 1 Flowchart of included patients. ROC, receiver operating characteristic.

incidence of placenta previa coexisting with placenta accreta spectrum (PAS) has risen rapidly as a consequence of the increase in cesarean delivery rates and the implementation of a two-child policy over the past decade (4).

PAS includes placenta creta, placenta increta, and placenta percreta (5). Patients with placenta previa with increta or percreta require cesarean section, during which blood loss can be severe. Therefore, there is an increased risk of related fatal maternal complications, such as disseminated intravascular coagulation, multisystem organ failure, and death $(6,7)$. Emergency hysterectomy is an essential measure to control severe intraoperative hemorrhage and reduce complications; however, it results in inevitable infertility $(8,9)$. In China, hysterectomy is not widely accepted by patients, who usually demand preservation of their uterus. Complications of placenta increta and percreta during cesarean section can be severe and even fatal. For patients with placenta increta or percreta, the risk prediction of adverse events and hemostatic measures are the primary points in clinical diagnosis and treatment.

Ultrasonography and magnetic resonance imaging (MRI) are considered to be the primary methods for the diagnosis of placenta increta and percreta, owing to their accuracy in detecting placental invasion $(10,11)$. However, there are no system-recognized evaluation methods to predict intraoperative massive blood loss due to placenta increta or percreta (5). Therefore, the primary aim of the present study was to determine high-risk factors and to develop and validate risk prediction models for intraoperative massive blood loss during cesarean section in patients with placenta previa with placenta increta or percreta.

We present the following article in accordance with the TRIPOD reporting checklist (available at http://dx.doi. org/10.21037/atm-20-5160).

\section{Methods}

Researchers may contact the corresponding authors for the data within the article for future analysis.

\section{Study design and participants}

The present study was a retrospective analysis of all placenta increta and percreta patients who attended or were referred to the Department of Obstetrics, Qilu Hospital of Shandong University, Jinan, China, with a subsequent terminated pregnancy between January 2016 and June 2019. A total of 179 patients who were admitted from January 2016 to December 2017 were selected and allocated to the development group, while 81 patients who were admitted between January 2018 and June 2019 were allocated to the validation group. All patients were diagnosed with placenta increta or percreta intraoperatively, either by an obstetrician or by histopathology. When no penetration of the placenta close to the serous layer and no or little vascularity of the uterine serosa-bladder wall interface were observed, the case was classified as creta. When increased vascularity of the uterine serosa-bladder wall interface, myometrial thinning of the anterior wall, and penetration of the placenta close to the serous layer were observed, the case was classified as increta. Based on this, when the placenta penetrated through the uterine surface, even invading into the bladder or other organs, the case was classified as percreta.

The inclusion criteria were as follows: (I) a history of cesarean section; (II) availability of B-scan ultrasonography examination; (III) singleton pregnancy; and (IV) patient request for fertility preservation. The exclusion criteria were preoperative coagulation disorders or blood system diseases. The flowchart for patient selection is shown in Figure 1.

The characteristics of patients prior to delivery were collected from medical records. Preoperative clinical features included age at delivery, gestational age, gravidity, parity, number of previous cesarean sections, history of dilatation and curettage of the uterus, gestational diabetes mellitus, hemoglobin, placenta previa classification, retroplacental myometrial thickness, vascular lacunae within 
the placenta, hypervascularity of the uterine-placental margin, irregularity of the uterine-bladder interface, hypervascularity of the uterine serosa-bladder wall interface, hypervascularity of the cervix, and emergency cesarean section. Surgical characteristics included preoperative balloon placement in the abdominal aorta (BPAA), B-Lynch suture, ligation of the ascending branch of the uterine artery, and tourniquet binding of the lower uterine segment.

No uniform criteria exist to define adverse pregnancy outcomes in placenta increta or percreta $(12,13)$. In the present study, we selected intraoperative blood loss $\geq 2,500 \mathrm{~mL}$ for each case as the major adverse maternal endpoint. Patients were followed up for 6 months after delivery. The study was conducted in accordance with the Declaration of Helsinki (as revised in 2013). The study was approved by the Ethical Committee of Qilu Hospital in Jinan, Shandong Province, China (No. 2019013) and individual consent for this retrospective analysis was waived.

\section{Statistical analysis}

Baseline characteristics of patients were expressed as descriptive statistics, and values are shown as medians (interquartile ranges) or $\mathrm{n}(\%)$. Continuous data were compared using the Mann-Whitney U-test, and classified data were analyzed with Pearson's $\chi^{2}$-test or Fisher's exact $\chi^{2}$-test. Univariate logistic regression analysis was used to identify predictors associated with adverse maternal outcomes. Variables with $\mathrm{P}<0.10$ were enrolled in the multivariate logistic regression analysis. Results are presented as odds ratios, $95 \%$ confidence intervals (CIs), and $\mathrm{P}$ value factors. Based on the regression coefficients of independent variables, individualized nomogram prediction models were established. Discriminative power and calibration of high-risk factors were tested through receiver operating characteristic (ROC) curve and calibration curve analyses. Decision curve analysis was applied to assess the performance of the predictive models.

SPSS version 24.0 (IBM, Armonk, New York, USA), and $\mathrm{R}$ software version 3.6.2 (http://cran.r-project.org) were used for the statistical analyses.

\section{Results}

Baseline characteristics of women with placenta increta or percreta are presented in Table 1. A total of 260 pregnant women diagnosed with placenta increta or percreta were included in our research. All patients had previously undergone at least one cesarean section and placenta previa. Twenty-eight (10.8\%) patients had an emergency cesarean section because of fetal distress, uterine contraction, and vaginal bleeding. Sixty-six (25.4\%) patients selected BPAA before cesarean section. Seventy-one $(27.3 \%)$ patients experienced massive bleeding during cesarean section $(\geq 2,500 \mathrm{~mL})$, and hysterectomy was performed in $8(3.1 \%)$ patients. The most common surgical complication was bladder repair $(n=20,7.7 \%)$. None of the patients died during the study.

The results of the univariable logistic regression analysis are presented in Table 2. Risk factors according to multivariate logistic regressions are shown in Table 3. Four independent risk factors were included in prediction model A: vascular lacunae within the placenta, hypervascularity of the uterine-placental margin, hypervascularity of the cervix, and emergency cesarean section $(\mathrm{P}<0.05)$. After the addition of cesarean section characteristics, six independent risk factors were included in prediction model B: vascular lacunae within the placenta, hypervascularity of the uterineplacental margin, hypervascularity of the uterine serosabladder wall interface, hypervascularity of the cervix, emergency cesarean section, and no preoperative BPAA $(\mathrm{P}<0.05)$. The nomogram prediction models are shown in Figure 2.

In the development group, the area under the ROC curve (AUC) of model A was 0.732 (95\% CI: 0.655-0.809), the sensitivity was $84.4 \%$, and the specificity was $48.5 \%$. The AUC of model B was 0.839 (95\% CI: 0.781-0.897), the sensitivity was $91.1 \%$, and the specificity was $67.2 \%$. The difference between the AUCs of models A and B was 0.107 $(\mathrm{Z}=3.786, \mathrm{P}<0.001)$ (Figure $3 A)$. In the validation group, the AUC of model A was 0.736 (95\% CI: 0.626-0.845), the sensitivity was $76.9 \%$, and the specificity was $60 \%$. The AUC of model B was 0.829 (95\% CI: 0.742-0.916), the sensitivity was $84.6 \%$, and the specificity was $69.1 \%$. The difference between the AUCs of models A and B was 0.093 $(\mathrm{Z}=2.653, \mathrm{P}=0.007)$ (Figure 3B). Therefore, model B had a better discriminative power than model A.

The calibration curves of both models in the development group are shown in Figure 3C,D, and the slope was 1.00 and 1.00 , respectively. The calibration curves in the validation group are shown in Figure $3 E, F$, and the slope was 0.72 and 0.68 in models $\mathrm{A}$ and $\mathrm{B}$, respectively. When the slope was closer to 1.00 , the prediction model had better calibration power.

Decision curves were used to evaluate the clinical utility of models A and B. In both the training and validation 
Table 1 Baseline characteristics

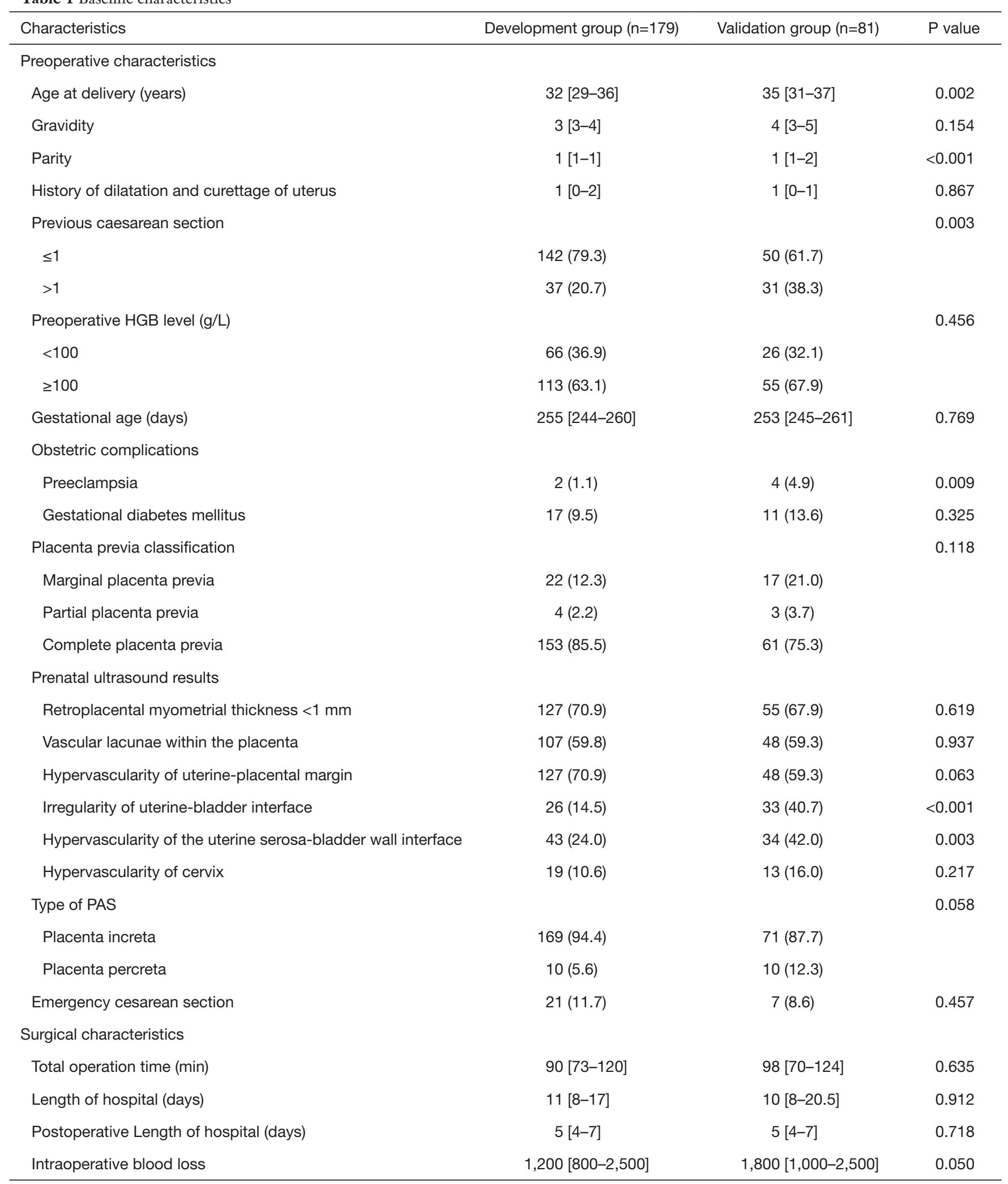

Table 1 (continued) 
Table 1 (continued)

\begin{tabular}{|c|c|c|c|}
\hline Characteristics & Development group $(n=179)$ & Validation group $(n=81)$ & $P$ value \\
\hline Units of PRBC transfused & $4[4-8]$ & $6[4-8]$ & 0.232 \\
\hline Preoperative BPAA & $46(25.7)$ & $20(24.7)$ & 0.863 \\
\hline B-Lynch suture & $36(20.1)$ & $40(49.4)$ & $<0.001$ \\
\hline Tourniquet binding the lower uterine segment & $23(12.8)$ & $18(22.2)$ & 0.055 \\
\hline Hysterectomy & $4(2.2)$ & $4(4.9)$ & 0.261 \\
\hline Bladder repair & $10(5.6)$ & $10(12.3)$ & 0.058 \\
\hline Systemic infections & $4(2.2)$ & $1(1.2)$ & 1.000 \\
\hline DVT or thrombotic requiring therapy & $0(0.0)$ & $1(1.2)$ & 0.312 \\
\hline DIC & $2(1.1)$ & $1(1.2)$ & 1.000 \\
\hline ICU & $2(1.1)$ & $2(2.5)$ & 0.591 \\
\hline
\end{tabular}

Values are median [interquartile range] or $n$ (\%). HGB, hemoglobin; PAS, placenta accreta spectrum; PRBC, packed red blood cells; BPAA, balloon placement of abdominal aorta; DIC, disseminated intravascular coagulation; DVT, deep vein thrombosis; ICU, intensive care unit.

groups, model B (black) showed a higher area under the decision curve than model A (red) (Figure 4).

\section{Discussion}

A retrospective study of 260 pregnant women with placenta increta or percreta who underwent cesarean section was constructed to establish two nomograms for predicting intraoperative massive blood loss. The model combining preoperative and surgical features showed better discrimination compared with the model combining preoperative features. The main findings of the present study were: (I) emergency cesarean section and ultrasound findings, including vascular lacunae within the placenta, hypervascularity of the uterine-placental margin, hypervascularity of the uterine serosa-bladder wall interface, and hypervascularity of the cervix are independently associated with massive blood loss; and (II) preoperative BPAA is a protective factor for massive blood loss. Therefore, preoperative ultrasound signs can help to identify high-risk patients early, and management during cesarean section can reduce intraoperative blood loss in patients with placenta increta or percreta. The combined model can be applied by obstetricians to enhance prenatal assessments of placenta increta or percreta patients, and to select the safest surgical treatment for these patients.

With the rising rates of cesarean section worldwide, from $6.7 \%$ in 1990 to $12.4 \%$ in 2010 , many studies have reported an increased incidence of placenta previa or placenta increta with massive hemorrhage $(14,15)$. Wright et al. found that previous cesarean deliveries had no association with massive blood loss $(\geq 5,000 \mathrm{~mL})(16)$. In the present study, the 75 th quantile of intraoperative blood loss was $2,500 \mathrm{~mL}$, and $71(27.3 \%)$ patients experienced this outcome; therefore, intraoperative blood loss $\geq 2,500 \mathrm{~mL}$ was taken as the adverse outcome. More than one previous cesarean section was not found to be a risk factor of intraoperative blood loss $\geq 2,500 \mathrm{~mL}$ in the multivariate logistic regression analysis. Blood transfusion support is challenging, for the average blood loss during a delivery complicated by PAS was from 2 to $5 \mathrm{~L}$ (17). Compared with placenta creta or increta, patients with placenta percreta are more likely to require additional blood products and have a higher incidence of urological complications, including ureteric injury and cystotomy (18). Therefore, preoperative evaluation of placenta increta or percreta is crucial for predicting peripartum outcomes (6).

Ultrasound and MRI are first-line methods for the 
Table 2 Univariate analysis in the development group

\begin{tabular}{|c|c|c|}
\hline Characteristics & OR $(95 \% \mathrm{Cl})$ & $P$ value \\
\hline Age (years) & $1.00(0.90-1.08)$ & 0.947 \\
\hline Gestational age (days) & $1.00(0.98-1.00)$ & 0.187 \\
\hline Caesarean section $>1$ & $1.59(0.72-3.50)$ & 0.253 \\
\hline GDM & $2.28(0.81-6.41)$ & 0.117 \\
\hline $\mathrm{HGB}<100 \mathrm{~g} / \mathrm{L}$ & $1.05(0.52-2.12)$ & 0.884 \\
\hline Placenta previa classification & $1.01(0.60-1.67)$ & 0.986 \\
\hline Retroplacental myometrial thinning $<1 \mathrm{~mm}$ & $1.36(0.63-2.95)$ & 0.433 \\
\hline Hypervascularity of the uterine serosa-bladder wall interface & $1.89(0.90-3.99)$ & 0.094 \\
\hline Hypervascularity of cervix & $2.42(0.91-6.46)$ & 0.078 \\
\hline Emergency cesarean section & $4.01(1.57-10.23)$ & 0.004 \\
\hline \multicolumn{3}{|l|}{ Surgical characteristics } \\
\hline No preoperative BPAA & $4.68(1.57-13.91)$ & 0.006 \\
\hline B-Lynch suture & $0.84(0.37-1.92)$ & 0.683 \\
\hline Ligation of ascending branch of uterine artery & $0.83(0.35-1.95)$ & 0.668 \\
\hline
\end{tabular}

GDM, gestational diabetes mellitus; HGB, hemoglobin; BPAA, balloon placement of abdominal aorta; OR, odds ratio; Cl, confidence interval.

Table 3 Multivariate logistic regression models in the development group

\begin{tabular}{|c|c|c|c|}
\hline Variables & Regression coefficients & OR $(95 \% \mathrm{Cl})$ & $P$ value \\
\hline \multicolumn{4}{|l|}{ Model A } \\
\hline Vascular lacunae within the placenta & 1.292 & $3.64(1.45-9.14)$ & 0.006 \\
\hline Hypervascularity of uterine-placental margin & 1.230 & $3.42(1.26-9.27)$ & 0.016 \\
\hline Hypervascularity of cervix & 1.155 & $3.17(1.08-9.33)$ & 0.036 \\
\hline \multicolumn{4}{|l|}{ Model B } \\
\hline Vascular lacunae within the placenta & 1.444 & $4.24(1.97-21.66)$ & 0.005 \\
\hline Hypervascularity of uterine-placental margin & 1.877 & $6.53(1.55-11.61)$ & 0.002 \\
\hline Hypervascularity of the uterine serosa-bladder wall interface & 1.337 & $3.81(1.23-11.77)$ & 0.020 \\
\hline
\end{tabular}

BPAA, balloon placement of abdominal aorta; OR, odds ratio; $\mathrm{Cl}$, confidence interval. 


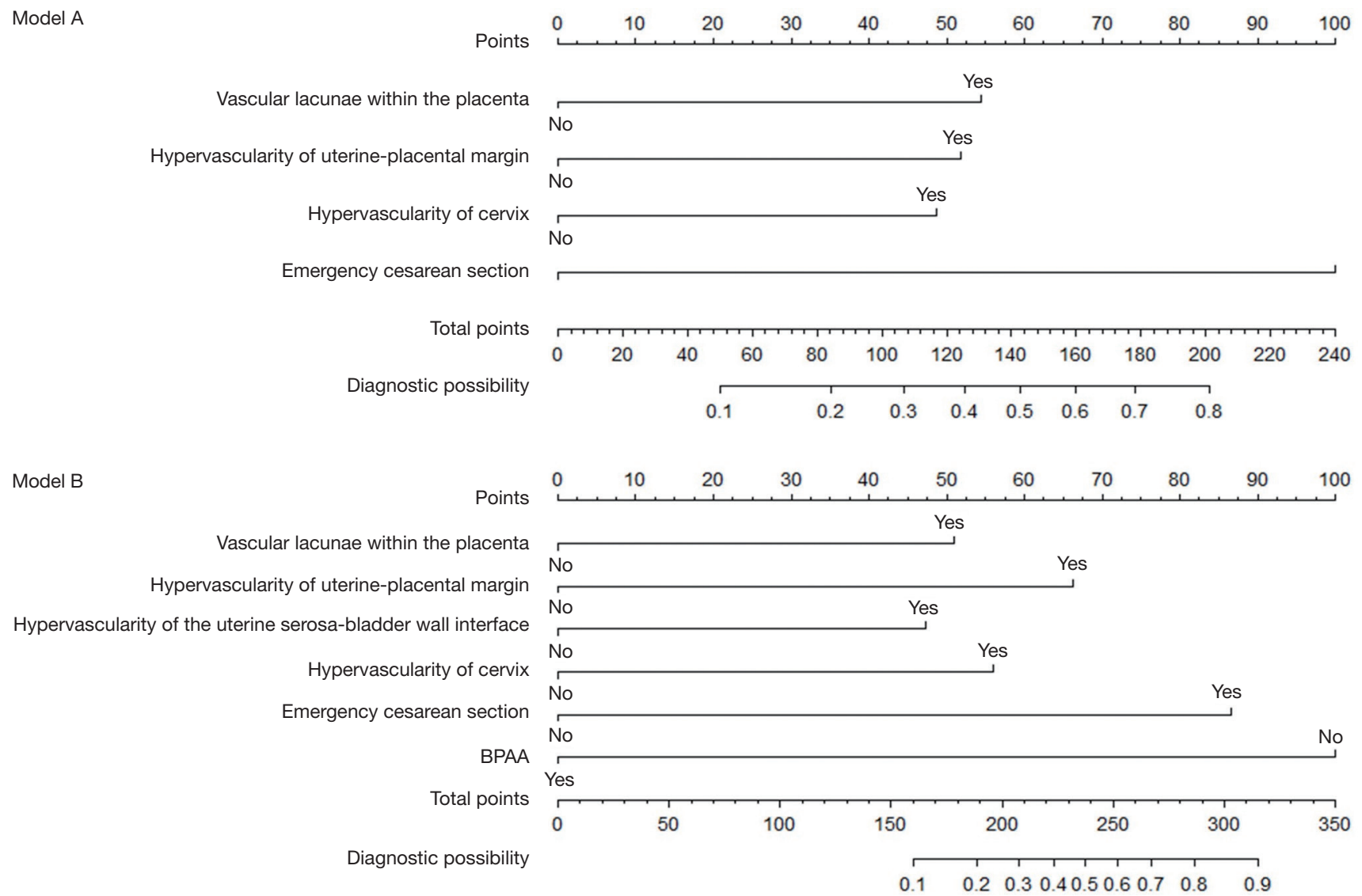

Figure 2 Nomograms to predict the probability of intraoperative massive blood loss in the patient with placenta increta or percreta. The nomogram can be applied by following procedures: (I) obtain the points corresponding to each predictor, (II) the sum of the points is recorded as the total score, and (III) the predicted risk corresponding to the total score is the probability of intraoperative blood loss $\geq 2,500 \mathrm{~mL}$ in placenta increta and percreta.

diagnosis of PAS, with reported sensitivity and specificity values of $97 \%$ and $97 \%$ for ultrasound and $94.4 \%$ and $84 \%$ for MRI, respectively $(10,11)$. Recently, Rac et al. and Bourgioti et al. established prediction models according to ultrasound and MRI findings to evaluate the type of placental invasion $(19,20)$. In their study, Collins et al. summarized the ultrasound signs used to diagnose PAS (21). However, no specific ultrasound sign or combination of ultrasound signs to determine the depth of placental villous implantation or to accurately distinguish PAS classifications has been found (22). This may be due to the various clinical diagnostic criteria of PAS. A detailed diagnosis of PAS is often not reported when specimens are not available for histopathological examination in cases of placental creta or conservative treatment (23). In the present study, we combined ultrasound signs with other clinical characteristics of patients to establish prediction models. MRI features were not included in the analysis for the majority of patients who had not been evaluated by MRI. Ultrasound signs of placenta increta or percreta patients were found to be associated with intraoperative blood loss in model B, improving the early identification of high-risk patients prior to surgery.

During surgery, drugs, sutures, vascular occlusion, and interventional radiology are often used to manage and prevent severe blood loss in patients with PAS (24-26). A few prospective and retrospective studies have evaluated the role of prophylactic BPAA to mitigate bleeding and avoid hysterectomy during surgery (26-28). In the present study, surgical features were included to establish model $\mathrm{B}$, and we found that BPAA can significantly diminish the amount of intraoperative blood loss. Complications are a significant concern of preoperative BPAA. Wu et al. reported that 2 of 230 cases developed vein thrombosis of 

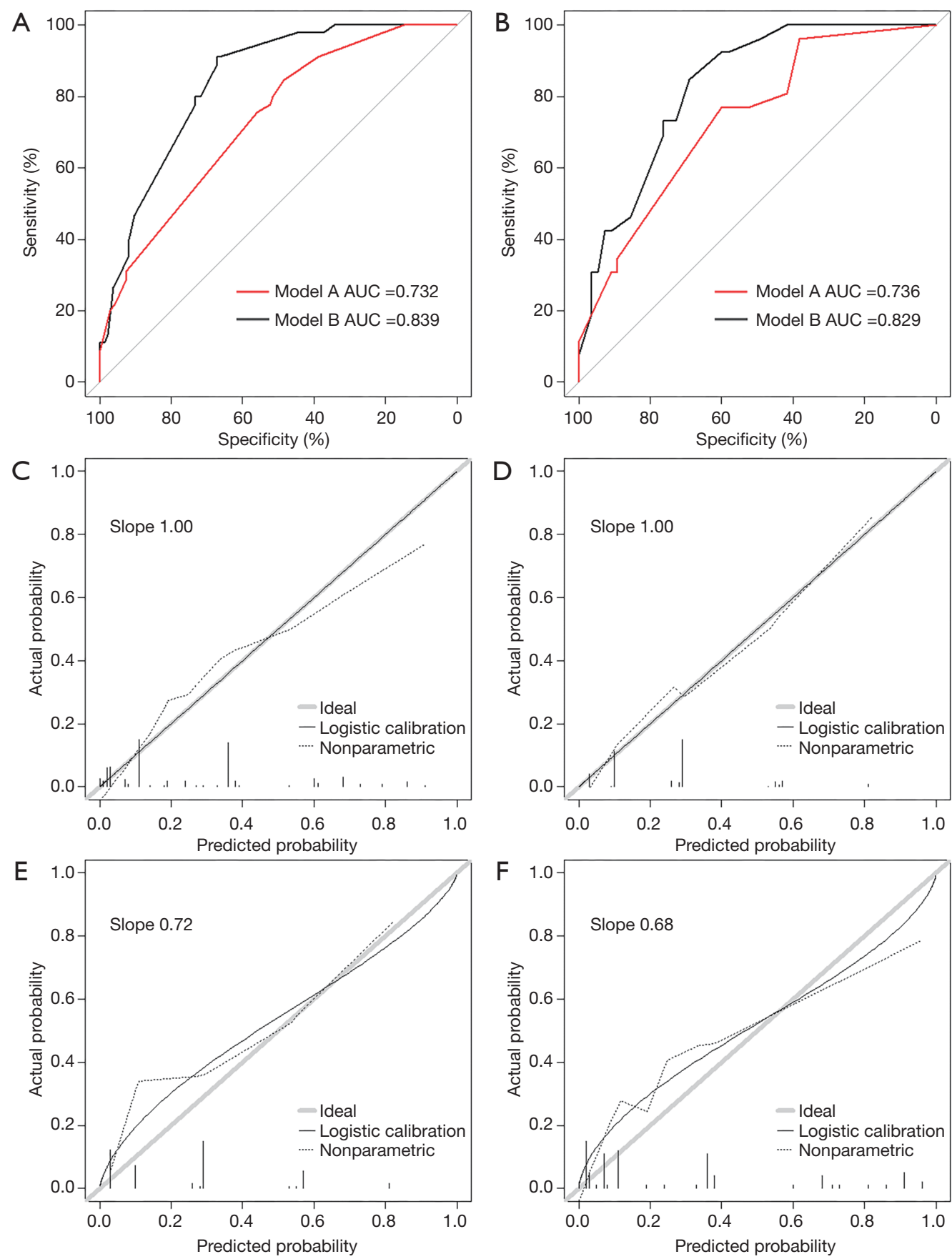

Figure 3 ROC curves and calibration plots. (A) Development group, AUC of model A is 0.732 (95\% CI: 0.655-0.809), AUC of model B is 0.839 (95\% CI: 0.781-0.897), $\mathrm{P}$ value is less than 0.001 (A vs. B); (B) validation group, AUC of model A is 0.736 (95\% CI: $0.626-0.845$ ), AUC of model B is 0.829 (95\% CI: 0.742-0.916), $\mathrm{P}$ value is 0.007 (A vs. B); (C) model A in development group, (D) model B in development group, (E) model A in validation group, (F) model B in validation group. Calibration curves were applied to evaluate the calibration of the models. The horizontal axis is the predicted probability provided by the model, and the vertical axis is the observed incidence of intraoperative blood loss $\geq 2,500 \mathrm{~mL}$. The 45-degree line is the actual probability. When the prediction probability of model is closer to the 45-dgree line, the prediction model has better calibration power. When the solid line is below the 45-dgree line, the prediction probability provided by the model is higher than the actual probability (overprediction); if the solid line is above the 45-dgree line, the prediction probability provided by the model is lower than the actual probability (underprediction). 

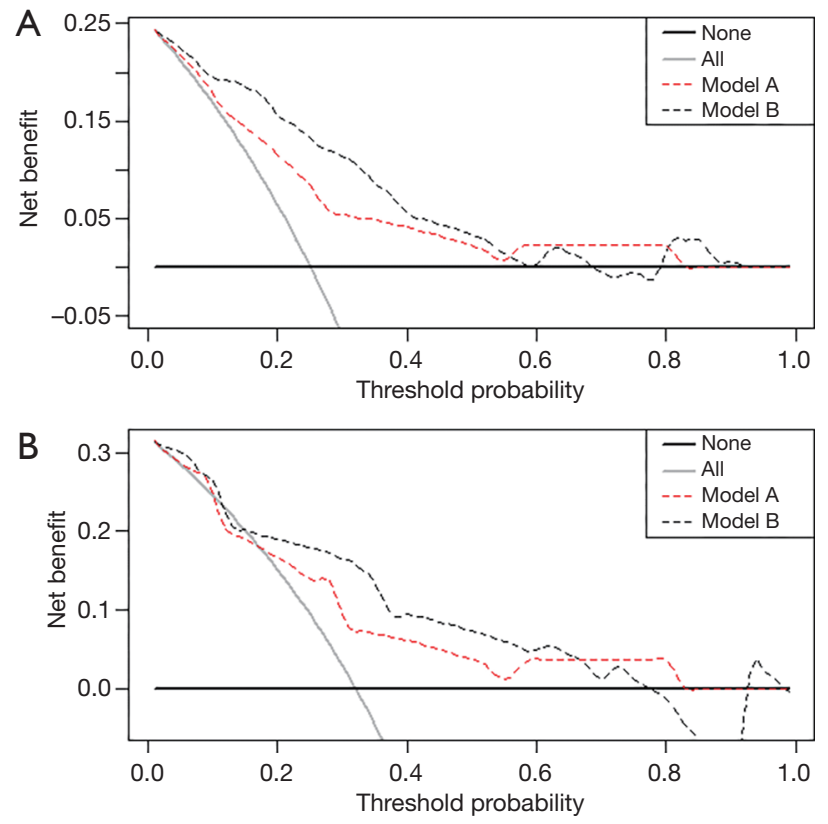

Figure 4 Decision curves. (A) Development group, (B) validation group. Draw the decision curve with the net benefit as vertical axis and the threshold probability as horizontal axis. The solid black line represents the net benefit when all patients are considered as not developing outcome (intraoperative blood loss $\geq 2,500 \mathrm{~mL}$ ). The solid grey line represents the net benefit when all patients are considered as developing outcome. The preferred model is the model with the highest net benefit.

the lower limbs (27). Zhu et al. reported one case that had abdominal aortic dissection complications (28). Injury of the vascular endothelium and occlusion-related thrombotic complications raise significant concerns for the safety of BPAA. In the present study, only one patient developed deep vein thrombosis of the lower limb after BPAA. Current studies have not sufficiently demonstrated the riskbenefit ratio of the use of abdominal aorta occlusion with balloon placement. Therefore, more well-designed studies are needed.

In the present study, gestational age was not found to be associated with massive blood loss; however, emergency cesarean section is a high-risk factor, and preoperative preparation is essential for these patients. As most patients request that their uterus not be removed, at our medical center, safe management to avoid massive blood loss is prioritized, including ultrasound screening and monitoring, hemostatic measures, and transfusion support.

A strength of the present study was the prenatal assessment strategy to identify high-risk factors associated with maternal adverse events in placenta previa with increta or percreta. A limitation of the study was that we only analyzed placenta increta or percreta in PAS, which usually causes intraoperative massive blood loss, and placenta creta was not included. In further research, we will explore all types of PAS, including placenta creta. If verified prospectively, our findings could be used to develop a specific procedure to identify and standardize the management of placenta increta or percreta patients, and ultimately improve pregnancy outcomes.

\section{Conclusions}

Risk prediction models established with preoperative and surgical characteristics can assist obstetricians to identify high-risk patients, develop treatment strategies to reduce the incidence of intraoperative massive blood loss, and further improve the prognosis of patients with placenta previa with increta or percreta.

\section{Acknowledgments}

The study was supported by Department of Obstetrics and Gynecology, Qilu Hospital, Shandong University, Jinan, Shandong, China.

Funding: This study was funded by Natural Science Foundation of Shandong Province in China (grant number ZR2015HM006).

\section{Footnote}

Reporting Checklist: The authors have completed the TRIPOD reporting checklist. Available at http://dx.doi. org/10.21037/atm-20-5160

Data Sharing Statement: Available at http://dx.doi. org/10.21037/atm-20-5160

Peer Review File: Available at http://dx.doi.org/10.21037/ atm-20-5160

Conflicts of Interest: All authors have completed the ICMJE uniform disclosure form (available at http://dx.doi. org/10.21037/atm-20-5160). The authors have no conflicts of interest to declare.

Ethical Statement: The authors are accountable for all 


\section{Page 10 of 11}

aspects of the work in ensuring that questions related to the accuracy or integrity of any part of the work are appropriately investigated and resolved. The study was conducted in accordance with the Declaration of Helsinki (as revised in 2013). The study was approved by the Ethical Committee of Qilu Hospital in Jinan, Shandong Province, China (No. 2019013) and individual consent for this retrospective analysis was waived.

Open Access Statement: This is an Open Access article distributed in accordance with the Creative Commons Attribution-NonCommercial-NoDerivs 4.0 International License (CC BY-NC-ND 4.0), which permits the noncommercial replication and distribution of the article with the strict proviso that no changes or edits are made and the original work is properly cited (including links to both the formal publication through the relevant DOI and the license). See: https://creativecommons.org/licenses/by-nc-nd/4.0/.

\section{References}

1. Downes KL, Hinkle SN, Sjaarda LA, et al. Previous prelabor or intrapartum cesarean delivery and risk of placenta previa. Am J Obstet Gynecol 2015;212:669.e1-6.

2. Thurn L, Lindqvist PG, Jakobsson M, et al. Abnormally invasive placenta-prevalence, risk factors and antenatal suspicion: results from a large population-based pregnancy cohort study in the Nordic countries. BJOG 2016;123:1348-55.

3. Sumigama S, Itakura A, Ota T, et al. Placenta previa increta/percreta in Japan: A retrospective study of ultrasound findings, management and clinical course. J Obstet Gynaecol Res 2007;33:606-11.

4. Zhang H, Dou R, Yang H, et al. Maternal and neonatal outcomes of placenta increta and percreta from a multicenter study in China. J Matern Fetal Neonatal Med 2019;32:2622-7.

5. Jauniaux E, Ayres-de-Campos D; FIGO Placenta Accreta Diagnosis and Management Expert Consensus Panel. FIGO consensus guidelines on placenta accreta spectrum disorders: Introduction. Int J Gynaecol Obstet 2018;140:261-4.

6. Silver RM. Abnormal placentation: Placenta previa, vasa previa and placenta accreta. Obstet Gynecol 2015;126:654-68.

7. Green L, Knight M, Seeney FM, et al. The epidemiology and outcomes of women with postpartum haemorrhage requiring massive transfusion with eight or more units

\section{Lu et al. Prediction of blood loss in placenta increta or percreta}

of red cells: a national cross-sectional study. BJOG 2016;123:2164-70.

8. Brookfield KF, Goodnough LT, Lyell DJ, et al. Perioperative and transfusion outcomes in women undergoing cesarean hysterectomy for abnormal placentation. Transfusion 2014;54:1530-6.

9. Jauniaux E, Alfirevic Z, Bhide AG, et al. Placenta Praevia and Placenta Accreta: Diagnosis and Management: Greentop Guideline No. 27a. BJOG 2019;126:e1-e48.

10. Jauniaux E, Bhide A. Prenatal ultrasound diagnosis and outcome of placenta previa accreta after cesarean delivery: a systematic review and meta-analysis. Am J Obstet Gynecol 2017;217:27-36.

11. D'Antonio F, Iacovella C, Palacios-Jaraquemada J, et al. Prenatal identification of invasive placentation using magnetic resonance imaging: Systematic review and metaanalysis. Ultrasound Obstet Gynecol 2014;44:8-16.

12. Souza JP, Cecatti JG, Haddad SM, et al. The WHO maternal near-miss approach and the maternal severity index model (MSI): tools for assessing the management of severe maternal morbidity. PLoS One 2012;7:e44129.

13. Jayaratnam S, Kua S, deCosta C, et al. Maternal 'near miss' collection at an Australian tertiary maternity hospital. BMC Pregnancy Childbirth 2018;18:221.

14. Betrán AP, Ye J, Moller AB, et al. The Increasing Trend in Caesarean Section Rates: Global, Regional and National Estimates: 1990-2014. PLoS One 2016;11:e0148343.

15. Guntupalli KK, Hall N, Karnad DR, et al. Critical illness in pregnancy: part I: an approach to a pregnant patient in the ICU and common obstetric disorders. Chest 2015;148:1093-104.

16. Wright JD, Pri-Paz S, Herzog TJ, et al. Predictors of massive blood loss in women with placenta accreta. Am J Obstet Gynecol 2011;205:38.e1-6.

17. DeSimone RA, Leung WK, Schwartz J. Transfusion Medicine in a Multidisciplinary Approach to Morbidly Adherent Placenta: Preparing for and Preventing the Worst. Transfus Med Rev 2018;32:244-8.

18. Marcellin L, Delorme P, Bonnet MP, et al. Placenta percreta is associated with more frequent severe maternal morbidity than placenta accreta. Am J Obstet Gynecol 2018;219:193.e1-9.

19. Rac MW, Dashe JS, Wells CE, et al. Ultrasound predictors of placental invasion: The Placenta Accreta Index. Am J Obstet Gynecol 2015;212:343.e1-7.

20. Bourgioti C, Zafeiropoulou K, Fotopoulos S, et al. MRI prognosticators for adverse maternal and neonatal clinical outcome in patients at high risk for placenta 
accreta spectrum (PAS) disorders. J Magn Reson Imaging 2019;50:602-18.

21. Collins SL, Ashcroft A, Braun T, et al. Proposal for standardized ultrasound descriptors of abnormally invasive placenta (AIP). Ultrasound Obstet Gynecol 2016;47:271-5.

22. Jauniaux E, Collins SL, Jurkovic D, et al. Accreta placentation: A systematic review of prenatal ultrasound imaging and grading of villous invasiveness. Am J Obstet Gynecol 2016;215:712-21.

23. Jauniaux E, Chantraine F, Silver RM, et al. FIGO consensus guidelines on placenta accreta spectrum disorders: Epidemiology. Int J Gynaecol Obstet 2018;140:265-73.

24. Chandraharan E, Krishna A. Diagnosis and management of postpartum haemorrhage. BMJ 2017;358:j3875.

25. Ordoñez CA, Manzano-Nunez R, Parra MW, et al.

Cite this article as: Lu R, Chu R, Gao N, Li G, Tang H, Zhou X, Lan X, Li S, Zhang X, Xu Y, Ma Y. Development and validation of nomograms for predicting blood loss in placenta previa with placenta increta or percreta. Ann Transl Med 2021;9(4):287. doi: 10.21037/atm-20-5160
Prophylactic use of resuscitative endovascular balloon occlusion of the aorta in women with abnormal placentation: A systematic review, meta-analysis, and case series. J Trauma Acute Care Surg 2018;84:809-18.

26. Silver RM, Branch DW. Placenta Accreta Spectrum. N Engl J Med 2018;378:1529-36.

27. Wu Q, Liu Z, Zhao X, et al. Outcome of Pregnancies After Balloon Occlusion of the Infrarenal Abdominal Aorta During Caesarean in 230 Patients With Placenta Praevia Accreta. Cardiovasc Intervent Radiol 2016;39:1573-9.

28. Zhu B, Yang K, Cai L. Discussion on the Timing of Balloon Occlusion of the Abdominal Aorta during a Caesarean Section in Patients with Pernicious Placenta Previa Complicated with Placenta Accreta. Biomed Res Int 2017;2017:8604849. 